[0212-7199 (2006) 23: 8; pp 387-388] ANALES DE MEDICINA INTERNA Copyright (C) 2006 ARAN EDICIONES, S.L.

AN. MED. INTERNA (Madrid) Vol. 23, N. ${ }^{\circ} 8$, pp. 387-388, 2006

\section{Fiebre de origen desconocido como presentación inicial de dos casos de carcinoma epidermoide de cérvix}

\author{
M. J. MOLINA GARRIDO, A. MORA RUFETE'1, C. GUILLÉN PONCE, \\ C. MARTÍNEZ Y SEVILA, A. CARRATO
}

Servicios de Oncología Médica y Medicina Interna. Hospital General Universitario de Elche. Alicante

\section{RESUMEN}

Presentamos dos casos de mujeres con FOD como primera manifestación de un cáncer de cérvix. En ambos casos, se hizo un despistaje exhaustivo de causas de fiebre. Los tratamientos antibióticos de amplio espectro (junto con tuberculostáticos, en la primera de las pacientes), no consiguieron erradicar la fiebre; los distintos tests de enfermedad autoinmune fueron negativos. Sólo las pruebas de imagen, en especial, la resonancia magnética nuclear, desvelaron que existía una lesión sospechosa, y fue la anatomía patológica la que corroboró la etiología de ambos procesos. El tratamiento del tumor (en ambos casos consistió en una cirugía radical) permitió la resolución de la fiebre. Se trata de dos casos representativos de fiebre etiquetados inicialmente como de origen desconocido, en los que un estudio exhaustivo reveló que esta era secundaria a cáncer epidermoide de cérvix, de los que no se dispone de literatura previa.

PALABRAS CLAVE: Fiebre de origen desconocido. Cáncer de cérvix. Resonancia magnética abdominal.
FEVER OF UNKNOWN ORIGIN AS FIRST MANIFESTATION OF TWO CASES OF CERVIX CARCINOMA

Molina Garrido MJ, Mora Rufete A, Guillén Ponce C, Martínez y Sevila C, Carrato A. Fiebre de origen desconocido como presentación inicial de dos casos de carcinoma epidermoide de cérvix. An Med Interna (Madrid) 2006; 23: X-X.

\section{INTRODUCCIÓN}

Se han descrito más de 200 causas de fiebre de origen desconocido (FOD). En los adultos, los tumores suelen ser la causa más frecuente, seguida por las infecciones, y posteriormente, por los procesos inflamatorios. En un $25 \%$ de los casos, no llega a conocerse el origen de la fiebre a pesar de un estudio completo.

\section{CASOS APORTADOS}

Caso 1. Paciente de 37 años de edad, sin antecedentes de interés. Presentaba fiebre con escalofríos de 3 meses de evolución, sin otra sintomatología ni foco aparente. La exploración física fue normal. La analítica mostró: VSG $94 \mathrm{~mm}, \mathrm{Hb} 8,5 \mathrm{~g} / \mathrm{dl}$, VCM 82,8 fl, leucocitos 19.630 (83,2\% de neutrófilos), plaquetas 504.000, fibrinógeno 1.237

\section{ASTRACT}

We present the case of two women with FUO as first manifestation of a cervical carcinoma. In both cases, wide spectrum antibiotic treatment (and tuberculostatic medication in the first woman) were not useful to stop fever. Autoimmune diseases tests were normal. Just image thecniques let us detect a suspicious lesion (specially, abdomino-pelvic magnetic resonance) and biopsy gave the definitive diagnosis. A radical surgery caused the fever resolution. These are two cases of cervical cancer as cause of fever of unknown origin and, at the moment, we have not found another cases in the literature. We should consider this kind of tumor as a possible origin of fever.

KEY WORDS: Fever of unknown origin. Cervix carcinoma. Abdominal magnetic resonance. 
29.690 (84\% de neutrófilos), plaquetas 555.000, VSG 114 mm, ferritina $1.467 \mathrm{ng} / \mathrm{ml}$, LDH $493 \mathrm{U} / \mathrm{L}$; PCR $450 \mathrm{mg} / \mathrm{L}$; marcadores tumorales: CA125 35 U/ml, CA15.3 43 U/ml, CA19.9 45,29 U/ml; hemocultivos y urocultivo negativos; serologías negativas; radiografía de tórax y enema opaco normales; se descartaron causas reumatológicas; TC abdómino-pélvico con cérvix uterino engrosado; exploración ginecológica: cérvix de aspecto tumoral con lesión en labio posterior y comisura izquierda. En la RMN abdomino-pélvica se apreciaba una masa de $5 \times 3,5 \times 3,5 \mathrm{~cm}$ en cérvix que invadía las vertientes anterior y posterior del cuerpo uterino, el tercio superior de la vagina y el parametrio izquierdo, hallazgos compatibles con un carcinoma de cérvix estadio IIb.

\section{DISCUSIÓN}

Se conocen más de 200 causas de fiebre de origen desconocido (FOD) y sólo en un $25 \%$ de los casos llega a conocerse la etiología de la misma (1). Recientemente, se ha publicado un posible algoritmo a seguir ante cualquier paciente con fiebre de origen desconocido, con la finalidad de optimizar las pruebas diagnósticas solicitadas (2). Los tumores representan un $9-20 \%$ del total de las causas de FOD (3), y en la actualidad, se consideran la primera causa de FOD en el adulto, seguido por los procesos infecciosos (4). En la literatura se han descrito distintas neoplasias como causantes de FOD, siendo las más frecuentes las hematológicas, y posteriormente, el sarcoma, el tumor cerebral, de colon, pancreático, renal y hepático (5); sin embargo, según la información de que disponemos tras la búsqueda bibliográfica hecha en Medline desde 1967, hasta la fecha, no se ha descrito ningún caso de carcinoma de cérvix manifestado como FOD. Por ello consideramos de interés comunicar dos casos de carcinoma epidermoide de cérvix que se presentaron inicialmente como fiebre de origen desconocido.

La FOD clásica fue definida por Petersdorf y Beeson (1961) (6) como aquel proceso de más de 3 semanas de duración con temperatura superior a $38,3^{\circ} \mathrm{C}$ en varias determinaciones, cuya causa permaneciera oculta tras una semana de estudios dirigidos. Durak y Street (7), en 1991, añadieron unas anotaciones a este concepto. En el año 2003, se modificó de nuevo esta definición, no siendo necesaria la permanencia del paciente en el hospital para poder diagnosticarlo de una FOD (8).

Cuando la FOD corresponde a un tumor oculto, suele exis- tir picos transitorios de fiebre acompañados frecuentemente por escalofríos, dato que estaba presente en la primera de nuestras pacientes. Los tumores que habitualmente se han asociado a la presencia de fiebre elevada son las leucemias agudas, los linfomas, los hepatomas, las metástasis hepáticas, las metástasis en el sistema nervioso central, el cáncer de mama inflamatorio, y los tumores renales. Por el contrario, el melanoma, el mieloma múltiple, los síndromes mieloproliferativos y las leucemias crónicas, es raro que produzcan picos febriles. Este hecho es aún más notable en el cáncer de mama, los sarcomas, los tumores gastrointestinales, el cáncer de páncreas, los tumores primarios del sistema nervioso central, el cáncer de próstata y el testicular, el cáncer de ovarios, los carcinomas escamosos y basales de la piel, y los tumores adrenales, que son tumores que rara vez se manifiestan como FOD (9).

Los tumores malignos suelen producir fiebre mediante la elaboración de citokinas, tales como el factor de necrosis tumoral, que producen una respuesta febril mediante su actuación sobre el núcleo preóptico del hipotálamo anterior. Generalmente, en aquellos tumores que no elaboran factor de necrosis tumoral, no suele producirse fiebre (9).

El cáncer de cérvix es la segunda neoplasia ginecológica más frecuente en las mujeres. El primer síntoma del cáncer de cérvix invasor es la hemorragia vaginal (10). La fiebre es un signo clínico que puede estar presente, aunque hasta ahora no se había descrito como la manifestación inicial del carcinoma de cérvix. Cuando esto ocurre, el tratamiento más adecuado de la fiebre consiste en tratar el propio tumor. En el primero de nuestros casos, el empleo de tuberculostáticos, corticoides y antibióticos, no consiguió erradicar la fiebre, hecho que sí ocurrió tras la cirugía; la segunda de las pacientes falleció por complicaciones propias de la estancia hospitalaria y de su situación basal.

Consideramos que los dos casos que presentamos son los primeros descritos en la literatura como carcinoma epidermoide de cérvix manifestado como FOD, por lo que queremos resaltar este hecho para que se tenga en cuenta dicho tumor dentro del despistaje de neoplasias causantes de FOD. También se pone de manifiesto la importancia de la exploración ginecológica en toda mujer con fiebre de origen desconocido, así como el protagonismo de la RMN en estos casos.

\section{Bibliografía}

1. Knockaert DC, Vanderschueren S, Blockmans D. Fever of unknown origin in adults: 40 years on. J Intern Med 2003; 253: 263-275.

2. Gaeta GB, Fusco FM and Nardiello S. Fever of unknown origin. Systematic review of the literature for 1995-2004. Nucl Med Commun 2006; 27: 205-211.

3. Arnow PM, Flaherty JP. Fever of unknown origin. Lancet 1997; 350: 575-80.

4. Cunha BA. Fever in malignant disorders. Infect Dis Pract 2004; 28.

5. Sorensen HT, Mellemkjaer L, Skriver MV, Johnsen SP, Norgärd B et al. Fever of unknown origin and cancer: a population-based study. Lancet Oncol 2005; 6: 851-55.
6. Petersdorf RG, Beeson PB. Fever of unexplained origin: report on 100 cases. Medicine (Baltimore) 1961; 40: 1-30.

7. Durack DT, Street AC. Fever of unknown origin-reexamined and redefinided. Curr Clin Top Infect Dis 1991; 11: 33-51.

8. Bryan CS. Fever of unknown origin: the evolving definition. Arch Intern Med 2003; 163: 1003-04.

9. Cunha BA, Mohan S, Parchuri S. Fever of unknown origin: Chronic lymphatic leukemia versus lymphoma (Richter's transformation). Heart Lung 2005; 34: 437-41.

10. Jordan LB, Monaghan H. Pathology of the cervix: recent developments. Clin Oncol (R Coll Radiol) 2004; 16: 248-54. 